\title{
TRANSPORTATION MODELLING IN PRACTICE: CONNECTING BASIC THEORY TO PRACTICE
}

\author{
WERNER HEYNS ${ }^{1}$ \& SIMON VAN JAARSVELD ${ }^{2}$ \\ ${ }^{1}$ Aurecon Pty Ltd, Pretoria, South Africa \\ ${ }^{2}$ Arup Pty Ltd, Johannesburg, South Africa
}

\begin{abstract}
Transportation planning and modelling can appear to be a complex process when first encountered. Transportation planning is a process that develops information to help make decisions on the future development and management of transportation systems. It involves the determination of the need for new or expanded highways, public transport systems, freight facilities, transportation terminals, their location, their capacity and the management of their demand. Typically, transportation planning involves a forecast of travel patterns and demand 15 to 25 years into the future with an aim to develop a future transportation system that will support spatial growth at that time. Travel demand occurs as a result of thousands of individual travellers making individual decisions on how, where and when to travel. These decisions are affected by many factors such as family situations, characteristics of the person making the trip, and the choices (destination, route and mode) available for the trip. Transportation modelling is used to forecast travel patterns and demand and includes numerous mathematical equations to simulate or represent how people travel.

This chapter explains how the process works, the assumptions made and the steps taken. It also provides an insight into how transportation modelling is applied in practice in demonstrating its role in the inter-relationship between spatial development and transport provision and planning.

Keywords: traffic, modelling, land use, transport integration, four step process, trip generation, trip distribution, mode split, trip assignment, land use, spatial development.
\end{abstract}

\section{INTRODUCTION}

The implementation of transport infrastructure and services are derived from transportation planning and modelling outputs. Transportation modelling and simulation as interface in the planning process plays an important role in enabling spatial development.

The models developed by transport planning professionals are simplified representations of reality which can be used to explore the consequences of particular policies, strategies and spatial changes. The models forecast transport demand based on expected spatial development, population and economic growth, which translates in the creation of additional trips on the road network requiring expansion or the provision of infrastructure for a variety of modes.

The travel forecasting process is at the heart of transportation planning (Goulias [1]). The process is used to estimate the number of trips that will be made on a transportation systems alternative at some future date. It involves a series of mathematical models that attempt to simulate human behaviour while traveling (Tri-County Regional Planning Commission [2]). The models include a sequence of steps that answer a series of questions about traveller decisions. Attempts are made to simulate all choices that travellers make in response to a given system of highways, public transport and policies. Many assumptions need to be made about how people make decisions, the factors they consider and how they react to a particular transportation alternative.

This chapter is broadly divided into two sections. The first 8 sub-sections provide basic theory and an overview of transportation modelling processes in general, considering the what, why, who and how of modelling and actual models used to forecast future travel. 
The second component of the chapter defines the practical application of modelling software, how it relates land use changes to infrastructure provision, the type of problems they seek to address, the decisions planning professionals have to make in deciding about the appropriate model in terms of applicability, costs and data requirements. Fig. 1 illustrates the components covered in this Chapter.

\section{LAND-USE AND TRANSPORT INTEGRATION}

The control and planning of land use is to a large extent the key to the control of both the demand for transport and its impact upon the environment. Although the inter-relationship between land use and transport is complex, it is well established. An inherent interaction or feedback process takes place between land use and transport, where the one influences and depends on the other. (American Association of State Highway and Transportation Officials [3], Toth [4]). Wegener [5] refers to the interaction as the land use transport feedback cycle, illustrated by Fig. 2.

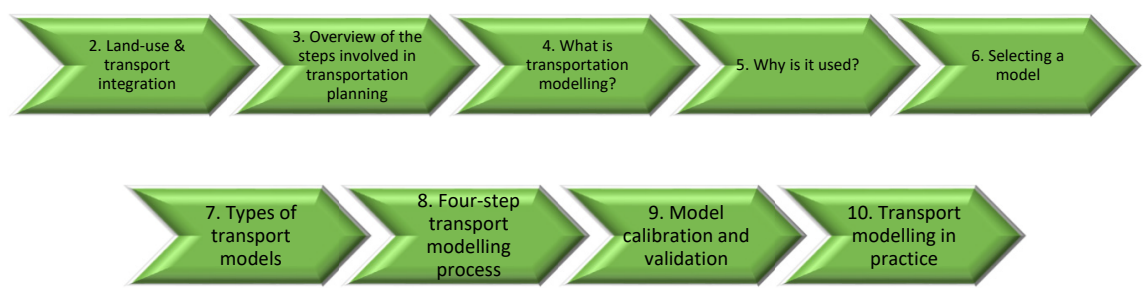

Figure 1: Components of transportation modelling examined.

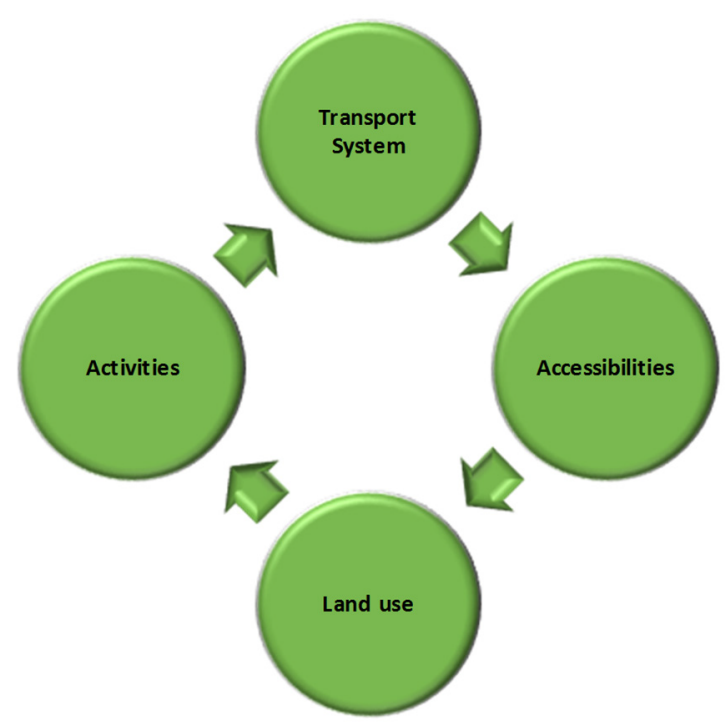

Figure 2: Land use transport feedback cycle. 
Starting at the bottom of the cycle (Land Use), the locations of population and employment determine the origins and destinations of most trips in travel models (Activities). Modelling the transportation system allows calculating accessibilities, which describe how well accessible a zone is to all other zones. Accessibility shapes land use, as both households and businesses search for locations that are - among other location factors - accessible.

Because of this interaction, it is important for transport and land use planning professionals to understand the likely inter-relationships and possible negative side effects when planning is not executed within an appropriate framework. For example, a land use policy aimed at encouraging urban sprawl, does little to promote shorter work-home trips, reduced travel cost and the financial requirements of operating and funding public transport. Counter intuitive and counterproductive land use and transport planning has serious long lasting consequences. In South African cities, the legacy of Apartheid is a good example demonstrating the transport inefficiencies resulting from inappropriate land policies, including:

- Misguided investment in transportation systems that prioritize high speed mobility over local accessibility (This places all other modes of travel at a disadvantage);

- Urban sprawl;

- Under provision of Non-Motorised Transport Infrastructure;

- Commuter public transport services focused on operating mainly during the morning and afternoon peak hours, with limited inter peak services;

- High travel cost (private and public transport) - mobility and accessibility is expensive and has a high economic and social cost;

- Rural communities often isolated and inaccessible, contributing to the inability to access opportunities and jobs resulting in perpetuated poverty.

Transportation modelling is a strong and helpful tool in the transport planner's tool kit that supports decisions making. It will not eradicate poor decision making or controversial land use policies, but some major advantages of using models in transport planning in supporting integration is that:

- it estimates likely land use transport interactions more quickly and at lower cost and risk than would be through implementation and monitoring or trial by error;

- it helps planners optimise the balance between land use provision and appropriate transport infrastructure and relevant modes to enable sustained economic growth;

- it defines the appropriate level of infrastructure required to support land use and spatial development;

- it defines the appropriate modes of transport to connect land use;

- it weighs up benefits/dis-benefits of various development scenarios; and

- it informs investment decisions by providing input into financial, economic, environmental and other relevant forms of appraisal.

3 OVERVIEW OF THE STEPS INVOLVED IN TRANSPORTATION PLANNING

Before exploring what transport modelling is, it is necessary to understand the broad steps involved in transportation planning as to understand how transportation modelling fits into the overall planning process.

Transportation planning goes through a basic sequence of steps. Several can take place simultaneously and it is not unusual to repeat some of the steps (United States Department of Transportation Federal Highway Authority of Transportation [6], [7]). Fig. 3 illustrates the broad overall planning process. 


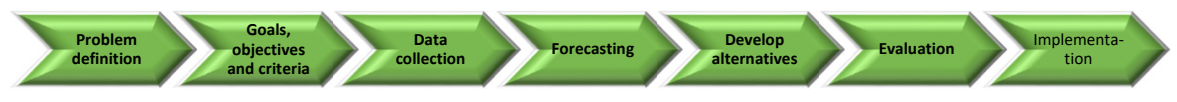

Figure 3: Basic steps in the transportation planning process.

- Problem definition: What is the key transportation, economic and land use issues and problems facing the community? This step may also involve definition of the size of an area to be studied, determination of the scope of the study and the establishment of a steering committee structure or forum to oversee the planning process.

- Define goals, objectives and criteria: A consensus should be developed by elected officials and citizens about the future of the community and its transportation system. Goals are developed for the quality of transportation service, environmental impacts and costs and are typically in conflict. A good planning effort will identify the trade-offs between these factors among alternatives in a clear, concise way to help make decisions.

- Data collection: Data must be compiled about the present status of the transportation system and its use. This could include traffic data, public transport ridership statistics, census information and interviews of households about their travel patterns. Data are also gathered on land use, development trends, environmental factors, and financial resources. This will help in problem definition and in developing methods to forecast future travel patterns. Good data are essential to the planning process. The statement 'garbage in/garbage out' applies in transportation planning. Without good data, the results of the planning process have little real meaning and can lead to wrong project selection and an inappropriate developmental direction for the region.

- Forecasts: Data from existing travel is used to make forecasts of future travel using travel demand models. This requires forecasts of future population, land use and economic conditions as well as understanding of how people make travel choices. Forecasting requires large amounts of data and is carried out under many assumptions which is based on benchmarking, sensitivity checks and follow up surveys to identify trends.

- Develop alternatives: Forecasts are used to determine the performance of alternative future land use and transportation systems. Alternatives normally include different land use and transportation patterns with different mixtures of highway and transit services and facilities. Since land use affects travel and travel affects land use, both must be considered.

- Evaluation: Results of forecasts are used to compare the performance of alternatives in meeting goals, objectives and criteria. This information may be extensively discussed by interested citizens, elected officials, different government agencies and the private sector. Ultimately decisions are made by appropriate elected or appointed officials and groups.

- Implementation of plans: Once decisions are made, plans should be further developed and refined for implementation. This may include more detailed analysis for design and evaluation following a similar process as above. 


\section{WHAT IS TRANSPORTATION MODELLING?}

Whilst many definitions exist defining transportation modelling it is primarily a mathematical tool using computer software to represent an actual transport system (the real world) to forecast travel patterns and flows between origins and destinations in geographic space by different modes (Victoria Transport Policy Institute [8]). The model output informs decisions about the type of infrastructure and transport modes to be implemented or operated in support of changing land use and travel patterns.

For example, when a new road is to be built, a transport model is usually developed to see how much traffic the road will carry, how wide it should be, where to include road intersections and defining the appropriate type of intersection control etc. After all, if you are able to save the cost of a slip road or the implementation of an additional lane on a highway the cost of developing and using the model is only a fraction. Because the investment requirement of transport infrastructure is very high and most often a burden on the fiscus clear advice is required to government or developers to provide value for money solutions. Transport models also sometimes cover public transport, walking and cycling and at the other end of the scale they cover air, sea and freight transport. These models may also include fares where the higher the fare the less people will use the infrastructure facility or mode of transport. This gives rise to the notion of elasticity in demand (Litman [9]), which is a relationship between a fare and the number of people who use a transport service or infrastructure.

\section{APPLICATION OF TRANSPORTATION MODELS}

Transportation models informs decisions about the type of infrastructure and transport modes to be implemented or operated in support of changing land use and travel patterns (Chandrasekaran [10]). Modelling helps to gain insight into complex situations by understanding simpler situations resembling them (Florian [11]). Professionals in transport and land use development also apply transportation models for some of the following reasons:

- Forecasting traffic flow;

- Assessing the outcomes of alternative land uses, infrastructure network and policy alternatives:

- For smaller areas - models are good for considering alternative designs (e.g. different sized roundabouts, intersection layouts etc.);

- For larger areas - to answer strategic questions such as whether or not to build new roads or implement urban pricing;

- Corridor studies;

- Supporting development control policy testing;

- Land-use transport interaction;

- Understanding travel behaviour;

- Establishing how to reduce traffic congestion;

- Establishing how to reduce fuel consumption;

- Establishing how to improve air quality;

- Optimizing the use of resources in building or maintaining transport systems; and

- Providing a platform for testing competing ideas and use in negotiations and decisionmaking.

Transportation models are used in a sequence of steps to answer a series of questions about future travel patterns that supports forecasting demand and ultimately planning decisions. 
The following questions follow a logical sequence and inform the steps in the modelling process:

1. What will our community/city/region look like in the future?

a. How many people will live there? (population forecasts)

b. What will they be doing? (economic forecasts)

c. Where will activities take place? (land use)

2. What are the travel patterns in the future?

a. How many trips will be made? (trip generation)

b. Where will the trips be? (trip distribution)

c. What modes will be used? (mode split)

d. What routes will be used? (traffic assignment)

e. What will be the effects of this travel? (impact analysis)

Ultimately transportation models should help the decision maker save money, define the appropriate level of transport investment required in support of spatial changes and implement value for money transport projects, systems and operations. Most modelling work is concerned with appraisal, in the broadest sense of the word, of alternative options or designs.

\section{SELECTING A MODEL}

When a range of models is available, the choice is influenced by the level of complexity of the problem being sought to be addressed. Models range in complexity from simple equations representing relationships which can be calculated on the back of an envelope, on a calculator in a spreadsheet or using powerful off the shelf (commercial) software programmes. The latter involving a large volume of coding to perform sophisticated mathematical functions or detailed simulations.

When selecting a model, the user should begin by defining the requirements of the exercise to be undertaken and the resources required. The requirements that should be defined include:

- What input variables are required, and to what level of detail?

- What output variables are required, at what level of detail and in what form (e.g. on screen results or graphics and results to "come alive" where the movement of cars are observed on screen)?

- Geographical coverage (will a single intersection be simulated, a number of intersections or an entire suburb?)

- Constraints of time and money;

- Is it possible to buy new commercially available software or should new software or processes be developed? and

- Determine whether new data can be collected.

The objective of defining the requirements for analysis to determine up front the most appropriate model for the task at hand, to save money and time and derive the right answer.

\section{TYPES OF TRANSPORTATION MODELS}

A variety of types of models is available to the analyst. The different traffic models each have its own pros and cons. The type of network under study and the traffic characteristics strictly influence the choice of the traffic model used for analysis. There is no one single model that can be used effectively for modelling traffic in all kinds of networks (Chandrasekaran [10]). For simplicity, two main groups exist: 
- Abstract mathematic models; and

- Simulation models.

The review provided below is not exhaustive and highlights the most common models within each group.

\subsection{Abstract mathematic models}

A number of abstract mathematical models exist including the following:

- Simple formulae: An equation of the general form $y=f(x)$ can be used to represent an empirically observed relationship between one variable $(x)$, which can be taken as given, and another $(y)$ which is to be predicted.

- Time series models: Many aspects of transport demand vary over time. For example, car ownership and total vehicle- kilometres have been rising almost continually over the past few decades, except for blips coinciding with fuel crises or economic recession. By plotting the relevant data over time, it becomes possible to deduce and underlying trend and or relationship. A prediction of future levels of travel demand can be made by extrapolating the trend into the future. Trend based forecasting is very popular because of its simplicity and because it is based on past evidence (Brockwell et al. [12]).

- Averaging and smoothing models: When no apparent trend data is available to support demand forecasting, simple arithmetic can be used to calculate an average or an exponentially smoothed prediction. Average forecasts are simply but very crude and may give a distorted view, exponential smoothing seek to overcome the crudeness, by giving weights to the most recent and trustworthy data.

- Regression analysis: This is the process of identifying the mathematical function (relationship) which best fits the observed data. The most common applications are in the prediction of car ownership, trip ends (numbers of origins and destinations at a given location) and trip volumes (in an area between a given pair of zones or along a link) (Jha et al. [13]). It is also used to predict waiting times at intersections, numbers of accidents or levels of pollution.

- Matrix estimation models: The cells of a demand matrix (known as an origin destination matrix) indicate the number of trips between each origin and destination pair, and the row and column totals indicate the total number of origins and destinations respectively in each zone. The matrix provides the overall picture of travel demand in a study area and is a key component in transport analyses.

- Elasticity models: When short term planning is undertaken, such that some influences can be considered constant, it is possible to specify a model which is solely concerned with the sensitivity of the forecast to key policy variables - known as elasticity modelling The elasticity of demand with respect to a certain variable is defined as the rate of change of demand with respect to that variable, normalized by the current levels of demand and the variable in question (Meyer and Miller [14]). Elasticity is thus a measure of sensitivity to change in system conditions (He and Zhao [15]). Its general form is:

$$
Y_{t}=Y_{t-1}\left\{1+E\left(X_{t}-X_{t-1}\right) / X_{t-1}\right\}
$$

where, $Y_{t}=$ quantity demand in year $t, X_{t}=$ value of a supply variable (e.g. price or journey time) in year $t$, and $E$ is the elasticity coefficient for $Y$ with respect to $X$. 
The elasticity coefficient should be calibrated on past data containing evidence of the marginal effect on $Y$ of a marginal change in $X$.

- Demand allocation - modelling the choice between alternatives: Trips are made as a result of choices made by travellers; choices between alternative modes of travel, alternative times of travel and alternative routes. Some of these choices are heavily constrained (e.g. if the traveller has no car available or if a public transport timetable provides only one service per day) while others may be made from a very extensive choice set. Different groups in the population have different constraints and those who have a very restricted choice set may be described as captives of a particular option. Even if they are not captive to one option some groups may have a strong preference for one option over the others; for example, high income travellers are likely to have a high value of time which causes them to prefer a fast and expensive option over a slower and cheaper one.

There is a wide and growing range of models which seek to predict travellers' choices among available options. They vary in sophistication from models which simply aim to reproduce an observed pattern to others which seek to replicate the underlying choice processes. An important concept underlying many of these models is market segmentation whereby separate forecasts are made for subgroups within the total population who, by virtue of their particular characteristics of car ownership, income, journey purpose etc., may be expected to respond to the choices available differently from other subgroups. Having made a forecast, for each subgroup, of the proportion of group members selecting each option, the overall forecast is simply achieved by weighting the proportions by the expected size of each subgroup in the forecast year's population.

\subsection{Simulation models}

Simulation models differ from abstract mathematical models in that they attempt to represent the dynamic evolution of some aspect of the transport system through an explicit representation of the behaviour of actors within it. Therefore, at a detailed scale, the performance of an intersection might be simulated by representing individual cars passing through it and the performance of a bus route might be simulated by representing the boarding and alighting of individual passengers at each stop. At a less detailed level, the development of a bus network might be predicted as the result of competition between different operating companies.

Another major difference between simulation and abstract modelling is that simulation modelling allows the analyst to develop a logic model of a very complex system by putting together a number of components which are themselves fairly well understood. It therefore becomes possible to develop a model of a complex system without having recourse to overarching theories about system dynamics or concepts such as equilibrium. When simulation is very detailed (zooming into fine detail analysis), it is sometimes referred to as micro-simulation, but the term is sometimes used interchangeably. When applied in traffic engineering the units are vehicles. When applied in travel behaviour the units are persons and households (Goulias [1]).

With cheaper, readily available commercial software programmes and ease of use and understanding opposed to abstract mathematical models, simulation modelling is undoubtedly the fastest growing area in modelling and are more often applied in solving transport and traffic problems.

Boxill and $\mathrm{Yu}[16]$ have classified simulation models into three categories: 
- Macroscopic;

- Mesoscopic models; or

- Microscopic models;

Simulation models can also be classified according to the nature of the network that they can be applied to, e.g. signalized networks, highway networks, and integrated networks or for specific purposes (Variable road pricing, etc.). Tables 1 to 3 illustrate a range of simulation modelling software programmes according to the classification by Boxill and $\mathrm{Yu}$ [16].

Table 1: Macroscopic models.

\begin{tabular}{|l|l|}
\hline Model & Classification \\
\hline ARTWORK & Highway networks \\
\hline AUTOS & Highway networks \\
\hline CORFLO & Integrated Networks \\
\hline FREFLO & Highway networks \\
\hline FREQ & Highway networks \\
\hline KRONOS & Unknown \\
\hline METACOR & Unknown \\
\hline METANET & Highway networks \\
\hline NETFLO & Signalized networks \\
\hline NETFLO 2 & Signalized networks \\
\hline NETVACI & Unknown \\
\hline PASSER-II & Signalized networks \\
\hline PASSER-IV & Signalized networks \\
\hline TRANSYT-7F & Signalized networks \\
\hline TRANSYT/10 & Signalized networks \\
\hline TEXAS & Signalized networks \\
\hline
\end{tabular}

Source: Boxill and $\mathrm{Yu}[16]$.

Table 2: Mesoscopic models.

\begin{tabular}{|l|l|}
\hline Model & Classification \\
\hline DYNAMIT & Integrated Networks \\
\hline DYNEMO & Integrated Networks \\
\hline DYNASMART & Integrated Networks \\
\hline
\end{tabular}

Source: Boxill and $\mathrm{Yu}[16]$. 
Table 3: Microscopic models.

\begin{tabular}{|c|c|c|c|}
\hline Model & Classification & Model & Classification \\
\hline AIMSUN & Integrated Networks & PLANSIM-T & Highway networks \\
\hline ANATOLL & Tolls & ROADSIM & Rural road network \\
\hline ARCADY & Signalized networks & SATURN & Integrated Networks \\
\hline AUTOBAHN & Highway networks & SHIVA & Other \\
\hline AVENUE & Signalized networks & SIGSIM & Signalized networks \\
\hline CARSIM & Signalized networks & SIMCO2 & Highway networks \\
\hline CASIMIR & Signalized networks & SIMDAC & Highway networks \\
\hline CONTRAM & Integrated Networks & SIMNET & Highway networks \\
\hline CORSIM & Integrated Networks & SIMTRAFFIC & Signalized networks \\
\hline DRACULA & Integrated Networks & SISTM & Highway networks \\
\hline FLEXYT II & Signalized networks & SITRA-B+ & Integrated Networks \\
\hline FOSIM & Highway networks & SIDRA & Integrated Networks \\
\hline FREEVU & Highway networks & SITRAS & Integrated Networks \\
\hline FRESIM & Highway networks & SMARTAHS & Unknown \\
\hline HIPERTRANS & Signalized networks & SMARTPATH & Highway networks \\
\hline HUTSIM & Signalized networks & SOUND & Integrated Networks \\
\hline ICARUS & Integrated Networks & SPEACS & Highway networks \\
\hline INTEGRATION & Integrated Networks & STEER & Highway networks \\
\hline INTRAS & Signalized networks & STREETSIM & Signalized networks \\
\hline JAM & No Available Data & TEXAS & Signalized networks \\
\hline MELROSE & Integrated Networks & TEXSIM & Signalized networks \\
\hline METROPOLIS & Highway networks & TRAFFICQ & Signalized networks \\
\hline MICROSIM & Signalized networks & TRANSIMS & Highway networks \\
\hline MICSTRAN & Signalized networks & TRGMSM & LRT \\
\hline MIMIC & Safety & TRITRAM & Highway networks \\
\hline MITSIM & Integrated Networks & THOREAU & Integrated Networks \\
\hline MIXIC & Highway networks & UTSS & Integrated Networks \\
\hline NEMIS & Integrated Networks & VEDENS & Signalized networks \\
\hline NETSIM & Integrated Networks & VISSIM & Integrated Networks \\
\hline OLSIM & Highway networks & VISSUM & Integrated Networks \\
\hline PADSIM & Highway networks & WATSIM & Integrated Networks \\
\hline PARAMICS & Integrated Networks & WEAVSIM & Integrated Networks \\
\hline PELOPS & Integrated Networks & & \\
\hline
\end{tabular}

Source: Boxill and $\mathrm{Yu}[16]$. 


\section{FOUR-STEP TRANSPORT MODELLING PROCESS}

Travel demand modelling was first developed in the late 1950s as a means to assist in highway planning. As the need to look at other problems and issues arose, the transportation modelling process was modified subsequently to add additional techniques to deal with these problems.

As a starting point one can ask, how is the city or region represented for computer analysis? Travel simulation require that an urban area or region be represented as a series of small geographic areas referred to as travel analysis zones (TAZs). Fig. 4 illustrates the travel analysis zones and links concept. Zones are characterized by their population, employment and other factors and are the places where trip making decisions are made (trip producers) and the trip need is met (trip attractors). Trip making is assumed to begin at the centre of activity in a zone (zone centroid).

Trips that are very short, that begin and end in a single zone (intrazonal trips) are usually not directly included in the forecasts. This limits the analysis of pedestrian and bicycle trips in the process. Zones can be as small as a single block but typically are 1/4 to one mile square in area. A planning study can easily use 500-2000 zones. A large number of zones will increase the accuracy of the forecasts but require more data and computer processing time. Zones tend to be small in areas of high population and larger in areas of low density and population. Internal zones are those within the study area while external zones are those outside of the study area. The study area should be large enough so that nearly all (over $90 \%$ ) of the trips begin and end within the study area. The trips that do not begin or end within the study area but travel through it is considered to be through traffic.
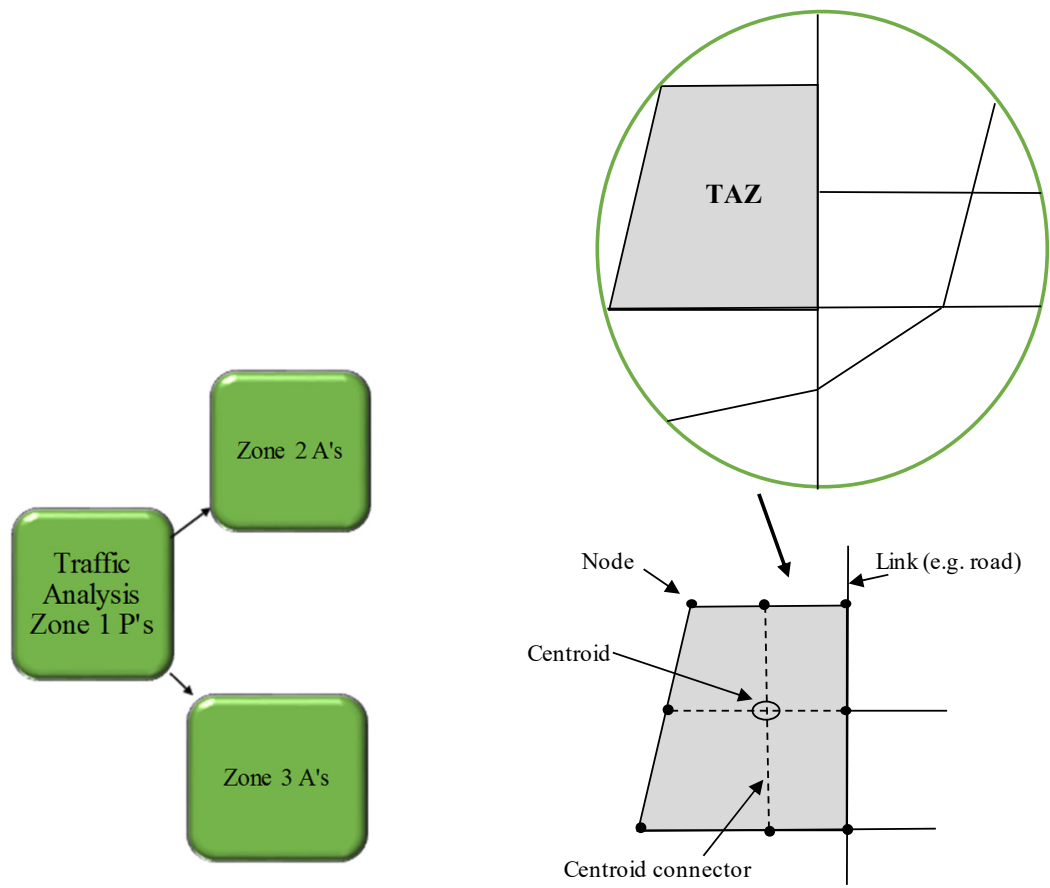

Figure 4: Travel analysis zones. 
The highway system and transit systems are represented as networks for computer analysis. Networks consist of links to represent segments of highways or public transport lines and nodes to represent intersections and other points on the network. Data for links includes travel times on the link, average speeds, capacity, and direction. Node data is more limited to information on which links connect to the node and the location of the node (coordinates). Node data could also include data on intersections to help calculate delay encountered at intersections.

The travel simulation process follows trips as they begin at a trip generation zone, move through a network of links and nodes and end at a trip attracting zone. The simulation process is known as the four-step process and includes (i) trip generation, (ii) trip distribution, (iii) mode split and (iv) traffic assignment (Tri-County Regional Planning Commission [2], Victoria Transport Policy Institute [8], Florian [11]).

\subsection{Trip generation}

The first step in travel forecasting is trip generation. In this step information from land use, population and economic forecasts are used to estimate how many trips will be made to and from each zone. This is carried out separately by trip purpose. Some of the trip purposes that could be used are: home based work trips (work trips that begin or end at home), home based shopping trips, home based other trips, school trips, non-home based trips (trips that neither begin or end at home), truck trips and taxi trips. Trips are calculated based on the characteristics of the zones. Trip productions are based on household characteristics such as the number of people in the household and the number of cars available. For example, a household with four people and two cars may be assumed to produce 3.00 work trips per day. Trip attractions are typically based on the level of employment in a zone. For example, a zone could be assumed to attract 1.32 home based work trips for every person employed in that zone. Trip generation uses trip rates that are averages for large segments of the study area. In South Africa, the following sources are used providing empirical estimates of trip generation per trip purpose or land use type:

- South African Trip Generation Rates Manual [17];

- Technical Methods for Highways (TMH) 17, Trip Data Manual [18]; and

- Institute of Transport Engineers (ITE), Trip Generation Manual [19].

\subsection{Trip distribution}

Trip generation only finds the number of trips that begin or end at a particular zone. The process of trip distribution links the trip ends to form an origin-destination pattern. Trip distribution is used to represent the process of destination choice, e.g. "I need to go shopping but where should I go to meet my shopping needs?" Trip distribution leads to a large increase in the amount of data which needs to be dealt with. Origin-destination tables are very large. For example, a 1200 zone study area would have 1,440,000 possible trip combinations in its O-D table for each trip purpose. The most commonly used procedure for predict trip distribution is gravity modelling. The gravity model takes the trips produced at one zone and distributes to other zones based on the size of the other zones (as measured by their trip attractions) and on the basis of the distance to other zones (see Fig. 5).

A zone with a large number of trip attractions (say a large shopping centre) will receive a greater number of distributed trips than one with a small trip attraction (a small shopping centre). 


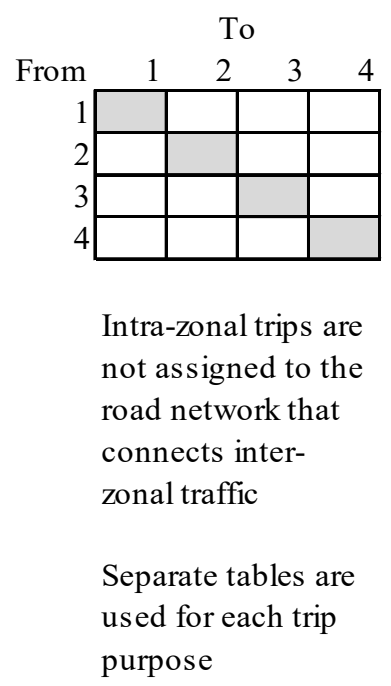

Figure 5: Origin-destination table.

Distance to possible destinations is the other factor used in the gravity model. The number of trips to a given destination decreases with the distance to the destination (it is inversely proportional). For example, you would expect more trips to a nearby shopping centre than one further away. The distance effect is found through a calibration process which gives travel times to destinations from the model similar to that found from field data. "Distance" can be measured in several ways. The simplest way this is carried out is to use car travel times between zones is as the measurement of distance.

Other ways might be to use a combination of car travel time and costs such as tolls as the measurement of distance. Still another way is to use a combination of public transport and car times and costs (composite cost). This method involves using a percentage of the car time and cost and a percentage of the public transport time/cost. Because of calculation procedures, the model must be used/run a number of times (iterated) in order to balance the trip numbers to match the initial values.

\subsection{Mode split}

Mode split or mode choice is one of the most critical parts of the demand modelling process. It is the step where trips between a given origin and destination are split into trips using public transport, trips by car pool or as car passengers and trips by car drivers - see Fig. 6 .

Calculations are conducted that compare the attractiveness of travel by different modes to determine their relative usage.

All proposals to improve public transport or to change the ease of using the cars are passed through the mode split/car occupancy process as part of their assessment and evaluation. It is important to understand what factors are used and how the process is conducted in order to plan, design and implement new systems of transportation.

Mode split is carried out by a comparison of the "disutility" of travel between two points for the different modes that are available. Disutility is a term used to represent a combination of the travel time, cost and convenience of a mode between an origin and a destination. 


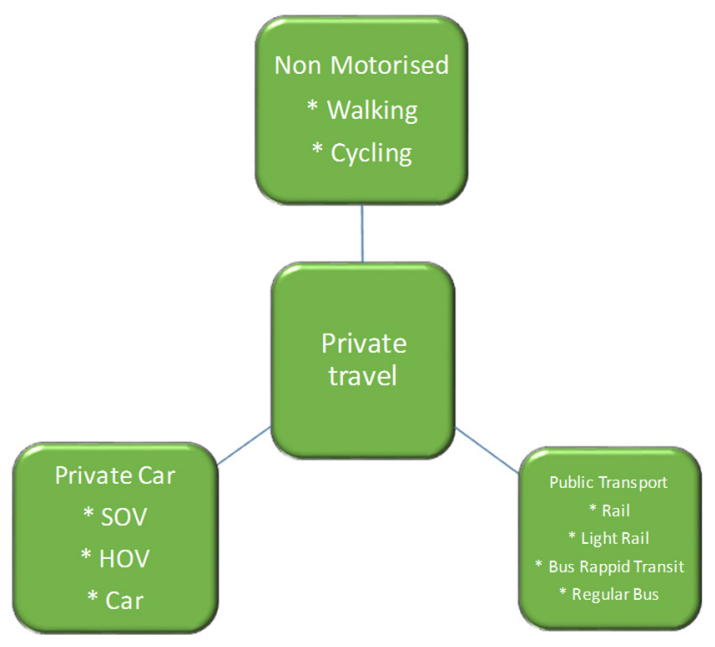

Figure 6: Mode split.

The disutility equations do not normally recognize differences within travel modes. A bus system and a rail system with the same time and cost characteristics will have the same disutility values. It is possible that mode bias factors could be different for different technologies, to represent a preference for light rail over bus, but this must be specifically included.

Once disutilities are known for the various choices between an origin and a destination, the trips are split among various modes based on the relative differences between disutilities. A large advantage will mean a high percentage for that mode. Splits are calculated to match splits found from actual traveller data. Sometimes a fixed percentage is used for the minimum transit use (percent captive users) to represent travellers who have no transport available or are unable to use a car for their trip.

Mode split and car occupancy analysis can be two separate steps or can be combined into a single step, depending on how a forecasting process is set up. In the simplest application, a highway/public transport split is made first which is followed by a split of car trips into car driver and car passenger trips. More complex analysis splits trips into multiple categories (single occupant auto (SOV), two-person car pool, 3-5-person car pool (HOV), van pool, local bus, express bus, etc.). Car occupancy analysis is often a highly simplified process which uses fixed car occupancy rates for a given trip purpose or for given household size and car ownership categories. This means that the forecasts of car-pooling are insensitive to changes in the cost of travel, the cost of parking, the presence of special programs to promote car-pooling, etc.

\subsection{Traffic assignment}

Once trips have been split into highway and public transport trips, the specific path that they use to travel from their origin to their destination must be found (Chiu et al. [20]). These trips are then assigned to that path in the step referred to as traffic assignment. Traffic assignment is the most time consuming and data intensive step in the process and is performed differently for highway trips and public transport trips. The process first involves the calculation of the 
shortest path from each origin to all destinations (usually the minimum time path is used). Trips for each O-D pair are then assigned to the links in the minimum path and the trips are added up for each link. The assigned trip volume is then compared to the capacity of the link to see if it is congested. If a link is congested the speed on the link needs to be reduced to result in a larger travel time on that link. When speeds and travel times are changed, the shortest path may change. Hence the whole process must be repeated many times (iterated) until there is an equilibrium between travel demand and travel supply.

Trips on congested links will be shifted to uncongested links until this equilibrium, condition occurs. There are a variety of ways in which the calculations are carried out to reach network equilibrium, in order to keep the computer time to a minimum. One way to get a feel for the accuracy of the models is to look at the resulting speeds on the network. These should be realistic after equilibrium.

Public transport trip assignment is carried out in a similar way except that public transport headways are adjusted rather than travel times. Public transport headways (minutes between consecutive vehicles) affect the capacity of the public transport route. Low headways mean that there is more frequent service and a greater number of vehicles. Public transport supply and demand are also recalculated to reach equilibrium between supply and demand.

It is important to understand the concept of equilibrium. If a highway or public transport route is congested during the peak hour, its excess trips will be shifted to alternative routes. If the alternative routes are also congested the final results will show congestion over a wide part of the network. In the real world, this congestion will eventually dissipate over time.

Another important step in assignment is the time of day analysis. Daily trip patterns need to be converted to peak time period traffic. A key assumption needed is the portion of daily travel that occurs during the peak period. This is normally used as a constant and conventional travel models have very limited capability to describe how travellers will shift their trips to less congested times of the day.

Some of the assumptions in traffic assignment are as follows:

- Delay occurs on links: Most traffic assignment procedures assume that delay occurs on the links rather than at intersections.

- Travel only occurs on the network: It is assumed that all trips begin and end at a single point in a zone (the centroids) and occurs only on the links included in the network. Not all roads streets are included in the network nor all possible trip beginning and end points included. The zone/network system is a simplification of reality.

- Capacities are simplified: To determine the capacity of roads and public transport systems requires a complex process of calculations that consider many factors. In most travel forecasts this is greatly simplified.

- Time of day variations: Traffic varies considerably throughout the day and during the week. The travel demand forecasts are made on a daily basis for a typical weekday and then converted to peak hour conditions. Daily trips are multiplied by a "hour adjustment factor", for example $10 \%$, to convert them to peak hour trips. The number assumed for this factor is very critical. A small variation, say plus or minus one percent, will make a large difference in the level of congestion that would be forecast on a network. Most models are unable to represent how travellers often cope with congestion by changing the time they make their trips.

- Emphasis on peak hour travel: As described above, forecasts are carried out for the peak hour. A forecast for the peak hour of the day does not provide any information on what is happening the other 23 hours of the day. The duration of congestion beyond the 
peak hour is not determined. In addition, travel forecasts are made for an "average or typical weekday". Variations in travel by time of year or day of the week are usually not considered.

\section{MODEL CALIBRATION AND VALIDATION}

Transportation models are aimed at reflecting an open system - the real world populated by people who are responding to influences that are constantly changing; do not always make rational decisions, and whose responses to influences affecting travel are not always the same. Since transportation models (and travel modellers) cannot be omniscient, there will always be missed information and abstractions resulting in less than perfect models.

Therefore, once the model is estimated and or set up using the four-step process, small adjustments are made to it until the model accurately replicates observed patterns and behaviours - referred to as calibration. Then the model is validated to a different set of observed data (for example, traffic counts) than was used for model estimation or calibration (United States Department of Transportation Federal Highway Administration [21], Wegmann and Everett [22]).

- Model calibration is the adjustment of constants and other model parameters in estimated or asserted models in an effort to make the models replicate observed data for a base year or otherwise produce more reasonable results;

- Model validation is the application of the calibrated models and comparison of the results against observed data; ideally, the observed data are not the same data used for model estimation or calibration.

Model calibration and validation generally occur in an iterative fashion. Model validation may reveal the need to return to the model estimation or model calibration steps. The application of the model using future year conditions requires that the model forecasts are reasonable and consistent with expectations and also might reveal a need to return to the model estimation or calibration step.

\section{TRANSPORTATION MODELLING IN PRACTICE}

Once a model has been developed, calibrated and validated it is ready for application, assessing policy decisions, testing the outcome of different scenarios or alternatives. This section outlines a number of examples where transportation modelling has been used to explore and solve "real life" problems - joining theory and practice. The examples demonstrate the use of models on a variety of levels. From the local level site specific at the one end to a regional level at the other end. To protect client confidentiality, street names and features that may identify buildings or land marks have been removed from the figures and images in this section.

The examples provided are presented in the form of seven case studies outlining the type of problem that was encountered, the type of modelling software used to address the problem and why and the outcome and or benefits of the modelling exercise.

\section{Case study 1}

The first case study relates to the local level - a site specific development, where a practical problem is considered. That is: "what is the sizing/vehicle capacity requirement of the site's vehicle entrance(s) to accommodate the forecasted vehicle demand?" When determining the number of vehicle entrances, there are two factors that determine ingress and egress capacity: 
(i) the number of lanes provided; and

(ii) the distance between the car park barrier/gates and the public road (also referred to as vehicle stacking distance).

If there are too little gate/lane capacity or the vehicle stacking distance is too short there is a risk that vehicles will spill over into the public road or stack on the public road causing congestion, road safety issues and traffic on the public road being negatively impacted (see Fig. 7 as a general illustration).

This is a very common problem and question when faced with new site developments. The success of the development depends on having sufficient access and the accesses operating optimally. If it does not the land owner/shop keeper may lose business as shoppers may consider going elsewhere if it is simply too difficult to access.

However, there is a very simple solution to solving the problem in isolation. A vehicle queuing model, determining the probability of vehicles that may stack, based on expected vehicle volumes and density, gate service levels and number of gates provided can be used as a suitable modelling tool to determine the optimum access requirements. It is inexpensive and data input requirements are limited.

\section{Case study 2}

The problem discussed in case study 1 can be expanded to a more complex problem where for example a development site has multiple accesses over which traffic is distributed into a large office building (as example). The question that arises is: "how do we design the car park optimally given a number of accesses and the inter-operability between the levels of structured or basement parking." This problem is compounded when the parking facility is a multi-storey (see Fig. 8) or underground car park with many parking levels accommodating a few thousands parking bays. The attraction of vehicles to each access is based on an 'attraction' factor, determined by the perceived access to parking bays. Whilst the distribution of vehicles around the site was determined during the trip distribution step in the four-step model, it did not address how the interaction of vehicles will occur once within the building and drivers are hunting parking spaces.

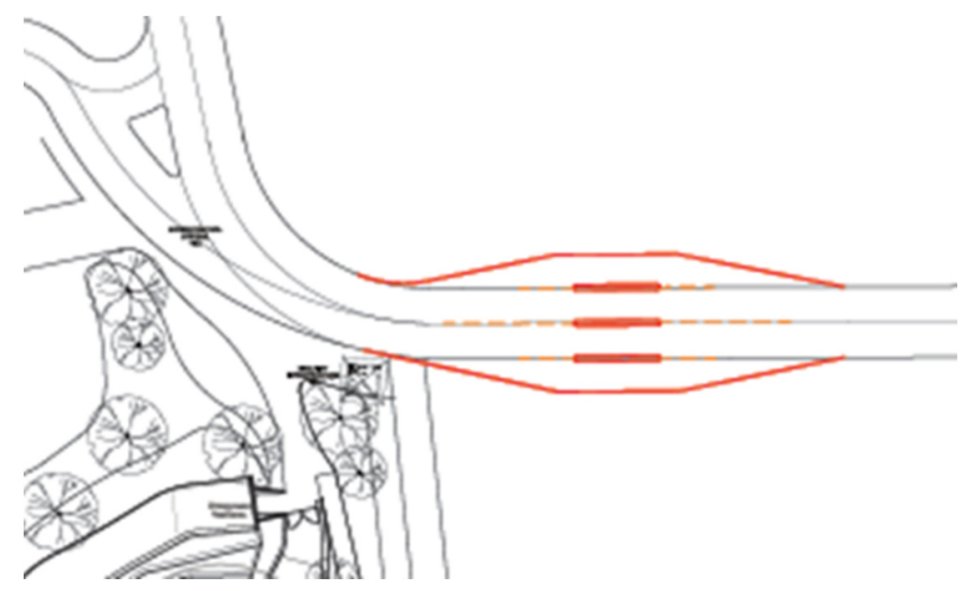

Figure 7: Determining vehicle access lane capacity. 


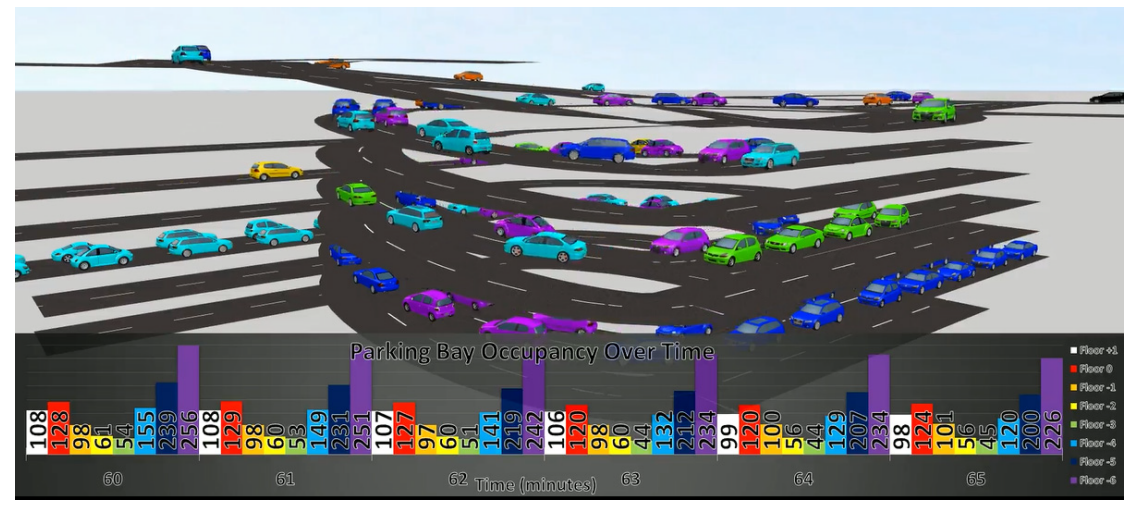

Figure 8: Structured parking.

Although using a spreadsheet and queuing analysis model in isolation will provide some clues to solve the problem it is incapable of solving the problem entirely due to the dynamic nature of the problem. Why? Because simple queuing analysis models consider one access problem at a time and not the inter relationship between different accesses along the same street or from different streets to the same site or building. The problem is further exacerbated where traffic streams at different accesses meet within the basement or structure parking as a result of random internal basement route choice.

To deal with this problem and the inter-related driver choices and behaviour dynamics, micro-simulation modelling with vehicle assignment functionality has proven to be a suitable modelling tool. Fig. 9 illustrates a screen shot of a micro simulation model being used to determine access requirements to a large-scale development.

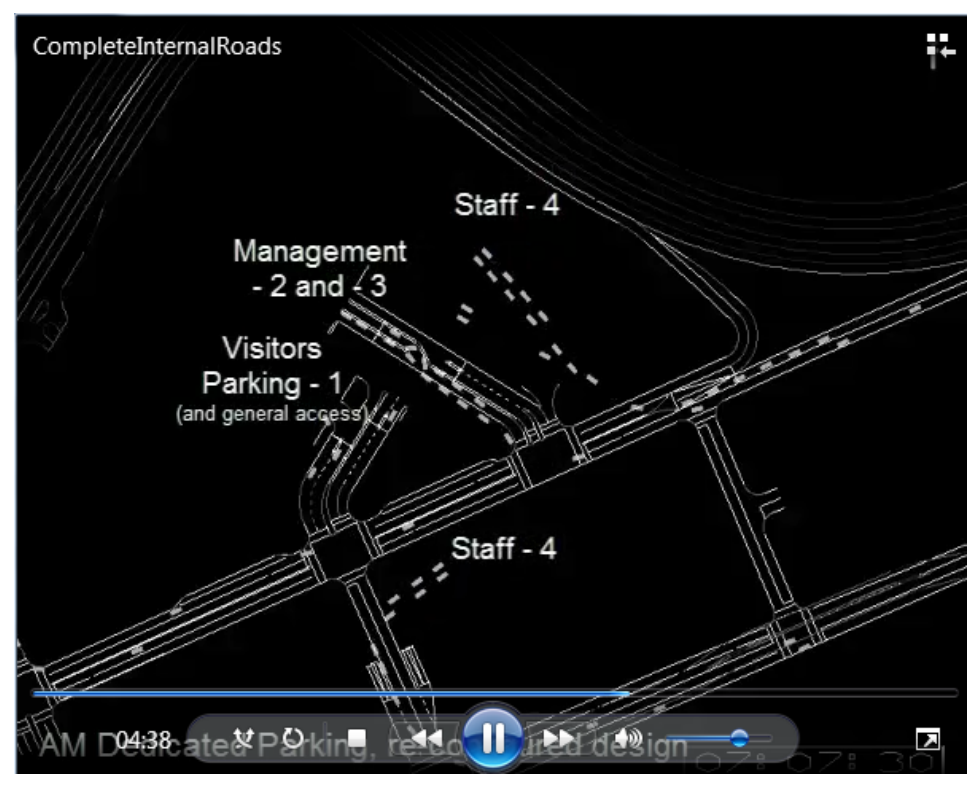

Figure 9: Micro-simulation modelling and parking design. 
One of the benefits of micro-simulation modelling is the visual presentation of the modelling results. Non-traffic engineers/transport planners are able to observe the 'logic' of how traffic patterns occur in and around the site/building and therefore reduce scepticism about whether the solution may be effective or not. The model is often used to demonstrate to clients and local authorities that a specific access strategy works optimally or that some other solution may be required to deal effectively with forecasted traffic volumes.

\section{Case study 3}

The previous two case studies examined access problems at the local or site specific level. However, traffic engineers and transport planners often have to assess the operation of intersections/interchanges on public roads, which are off site. Typically, such assessments are necessary as part of Traffic Impact Assessment studies or Environmental Impact Assessments. The challenges/problems faced here include some of the following:

- What is the most suitable intersection control type where two roads cross?

- What is the most appropriate configuration for an intersection?

- How much lane capacity is adequate?

- What geometry is suitable (e.g. grade separate or at grade)?

- How will a particular intersection operate in terms of Level of Service (LOS) and delay in a future scenario given a certain volume of traffic?

When intersections are considered in isolation (where interaction between intersections are not important for the purpose of the exercise), deterministic models such as SIDRA, Oscady, Picady and Arcady are used. These models are reasonable inexpensive to operate and data input requirements are limited. Existing and expected traffic volumes at an intersection can be modelled assuming different intersection types and lanes or reconfigurations can be added until a geometrical configuration is obtained that adheres to acceptable design and operational standards. The model output in the form of LOS, vehicle delay and queue lengths will inform how to conceptually design an intersection to a geometrical optimal layout. A variety of Traffic Engineering Manuals and guidelines exist defining the appropriate and or acceptable requirements and specification to render the intersections' operating level acceptable.

Typical requirements and specification include limited vehicle delay, limited vehicle queuing and a Volume/Capacity ratio that is less than 0.95 (see Fig. 10).

Finding the appropriate intersection control type is an iterative process. The process that would be typically followed is to test the most simplistic intersection type first given a specific volume of traffic. A "build up" process is then followed. For example, the following intersection types could be assessed sequentially for optimal suitability until an intersection type is identified that satisfies guidelines, specifications and requirements:

- Give-way

- Three or four-way stop

- Roundabout/traffic circle

- Signalisation

- Grade separation

As each intersection type is considered, intersection specification is changed until an optimal layout/geometry is determined. For example, lanes can be added to accommodate additional through or turning movements. Traffic circles or roundabouts however presents issues of its 


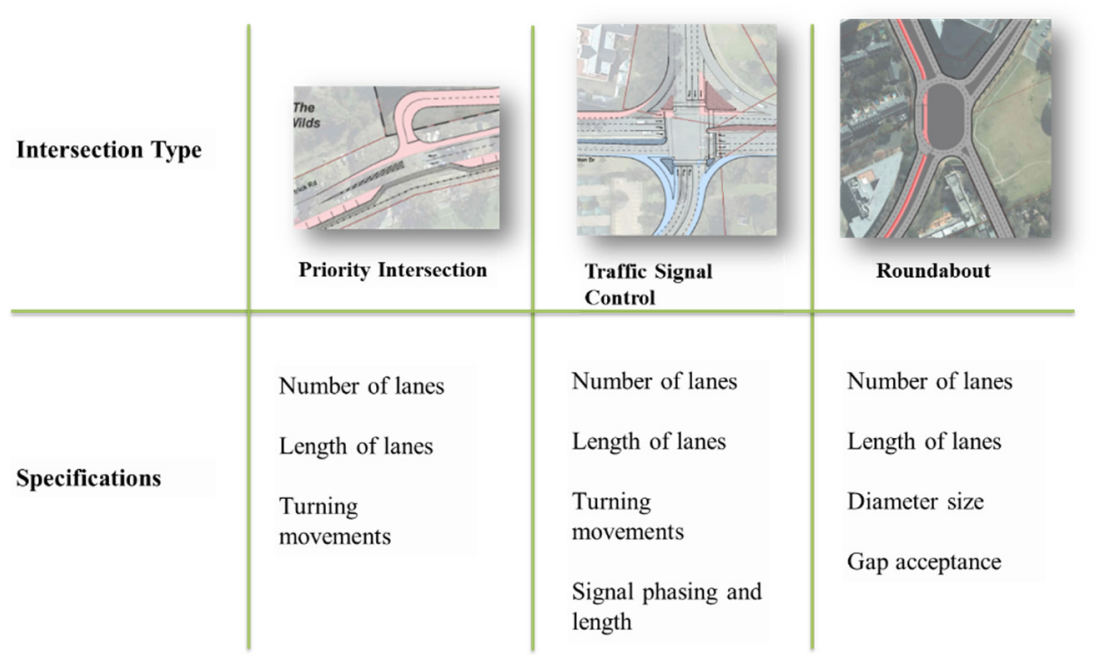

Figure 10: Requirements and specification for different intersection control types.

own including large land or space requirements and pedestrians having difficulty crossing from one side of the road to the other. Another factor to be considered is the cost of implementing the intersections. The more complex the intersection the higher the cost. Therefore, the decision about which intersection is the most appropriate depends on a variety of factors that cannot be fully addressed by transportation modelling alone. The most suitable intersection type is often a result of balancing different needs, consideration and stakeholder requirements.

\section{Case study 4}

The previous case study focused on defining the most appropriate intersection control type and geometrical layout of an intersection. However, intersections form part of a road network comprising a variety of roads intersecting with one another and their impact/influence on each other. Traffic flow at one intersection influences traffic flow at the next intersection (particularly in urban areas) and therefore it may not be realistic to design or assess intersections in isolation (particularly when the objective is to define the types of intersection control for a large area). Signal controlled intersections provides the opportunity to synchronise the operation of a series of intersections with one another allowing a platoon of vehicles arriving at a certain speed to be able to reach each intersection during the green phase and therefore not having to stop at any of the intersections. This is made possible by including an offset time equal to the anticipated time that it takes to travel from one intersection to another in the entire traffic phase.

The modelling software used for this operation would therefore need to be able to link one intersection to another and deterministic models that views intersections in isolation would not be able to provide this function. Fig. 11 illustrates a progression analysis output diagram for a series of three intersections from Transyt software. Transyt is particularly useful in signal optimisation and is reasonably inexpensive and data input requirements are limited. Typical data input requirements include intersections count data, queue length data and signal phasing plans. 


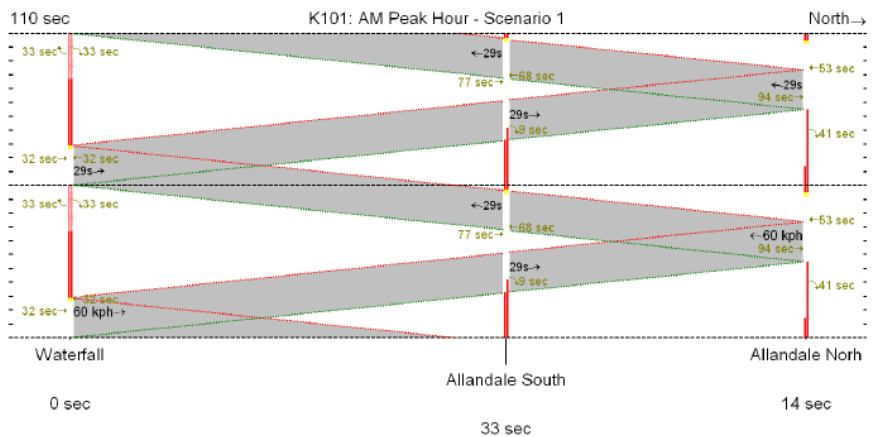

Figure 11: Transyt output.

\section{Case study 5}

In nodes or business districts where significant development growth occurs on multiple sites, a network based approach to transport planning is considered. The transportation model used should be able to assign traffic to the road network based on an estimated travel cost over a sufficiently wide enough area. The development of such a model includes an iterative process requiring multiple computations, which is too time consuming and repetitive to be calculated on paper or by using a spreadsheet.

For large-scale development or when having to present a region or urban node by a transportation model consisting of a vast network of road links and intersections affecting being interconnected and affecting one another a microscopic model is suitable, e.g. SATURN. These model types are expensive to develop, are traffic data hungry and take a lot of time to calibrate and validate.

The model would be developed as described in section 8 . For example, the area would be divided into travel analysis zones and for each the number of trips ending in it or generated by it would be identified. Road links and intersection capacity will be coded and act as the potential pathways for trips to find their destination. The model will assign trips to the route that shows the least resistance or in other words takes the least time for a trip to be made between two points or nodes. The images below shows assigned traffic volumes for modelled areas in Johannesburg using SATURN software (see Fig. 12).
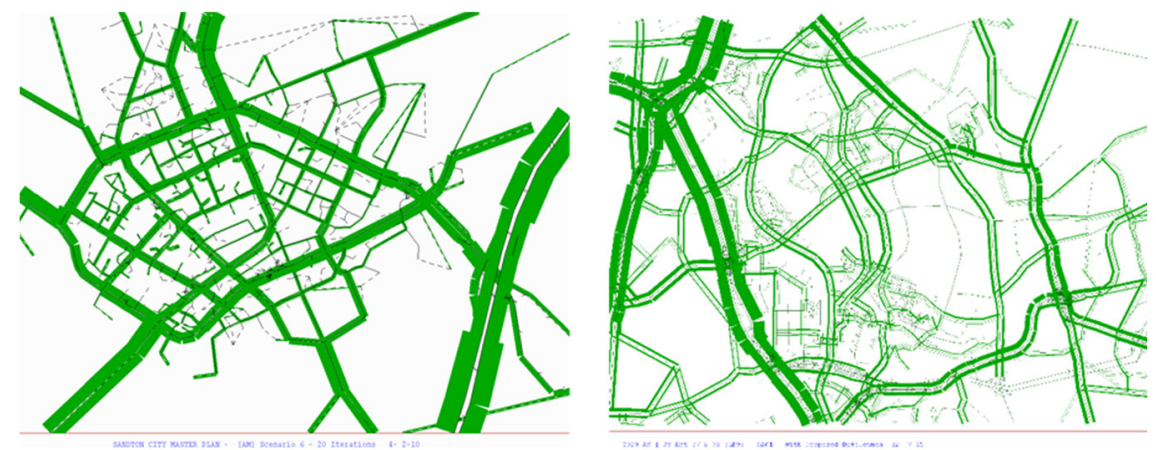

Figure 12: SATURN output. 


\section{Case study 6}

The traffic engineer or transport planner often has to decide what transportation infrastructure would be most beneficial within a road network given specific traffic volumes and characterises. Large scale infrastructure such as new interchanges, major roads and or bridges has far reaching traffic impacts and costs. For instance, drivers living up to say 15 to $20 \mathrm{~km}$ from a major road network might decide to change their travel behaviour due to new road infrastructure provided elsewhere or the lack of it to improve their travel mobility.

Fig. 13 illustrates large scale infrastructure proposed at an existing grade separated interchange. Again, microscopic modelling was carried out using SATURN software to determine which of a number of geometrical options would produce the optimal level of benefit to the surrounding area and whilst maintaining the mobility function of the road network. In Fig. 13 below it was considered that the provision of the southern interchange provides a new route option, while the northern interchange only increases existing capacity on an established route. The new route option dramatically changes travel patterns and relieves road infrastructure currently operating under tremendous pressure. This infrastructure was considered to be the most beneficial and was recommended for implementation.

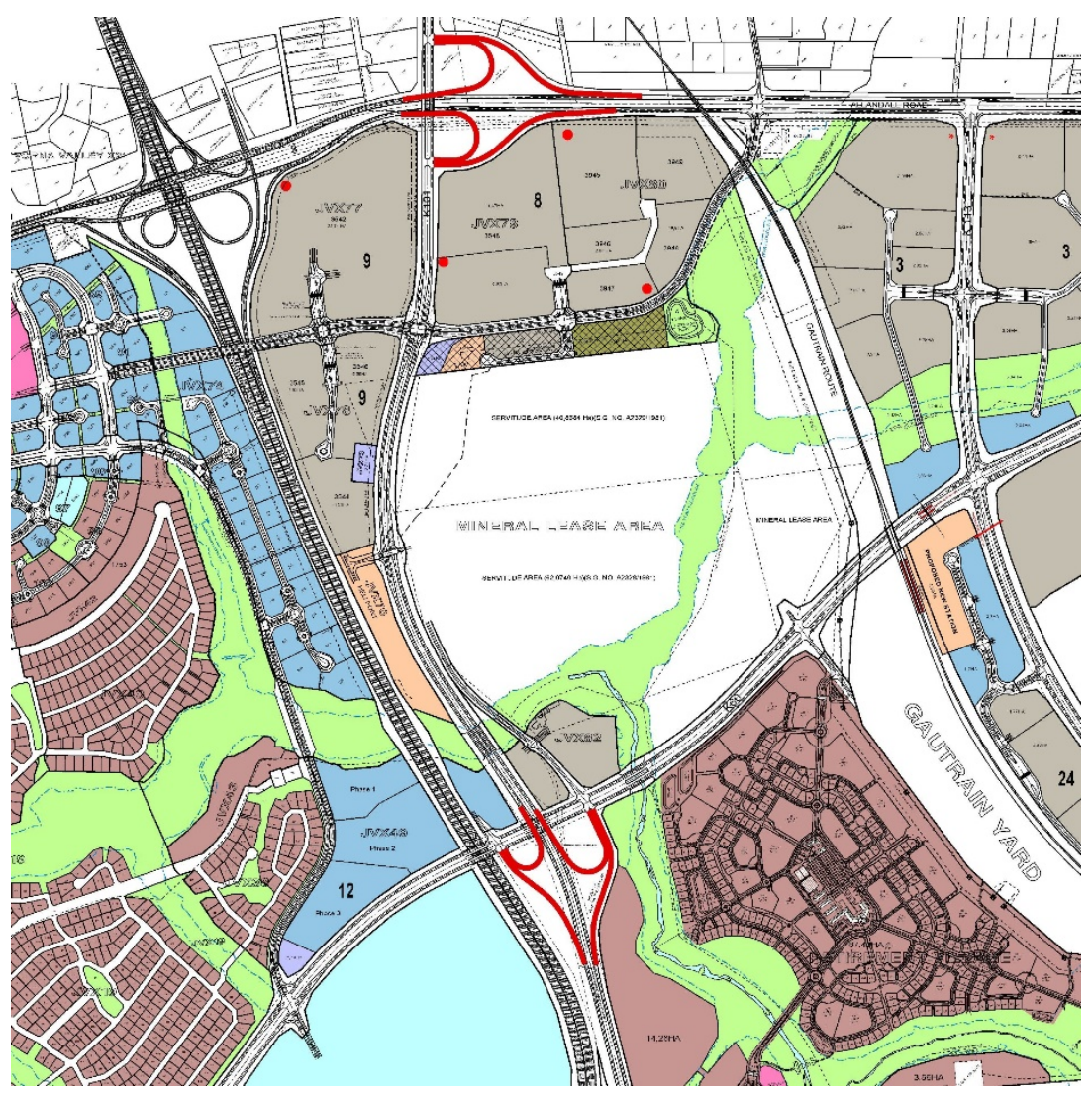

Figure 13: Large-scale infrastructure assessment. 


\section{Case study 7}

At a strategic planning level decisions have to be made on regional, provincial and national levels to determine the impact of long distance routes and other transport infrastructure and the interaction between them. The type of route infrastructure and possible public transport systems involved has huge capital costs and the benefits of its provision need to be understood well by decision-makers. To support the decision-making process travel demand models are often used to demonstrate the future expected utilisation of proposed infrastructure. Such benefits are converted into social benefit i.e. travel time savings, new employment opportunities and development opportunities to help decide among different options.

Fig. 14 represents a snapshot of an EMME model, a microscopic travel demand model. Whilst SATURN reviews the road network in more detail by considering for example number of lanes on road links and exact intersection configurations, EMME considers each road only as a route option with a generalised capacity. It therefore determines long distance travel desire lines within a region and allocates traffic accordingly but also considering the routes of least resistance and is therefore employed given its strategic geographical nature advantage.

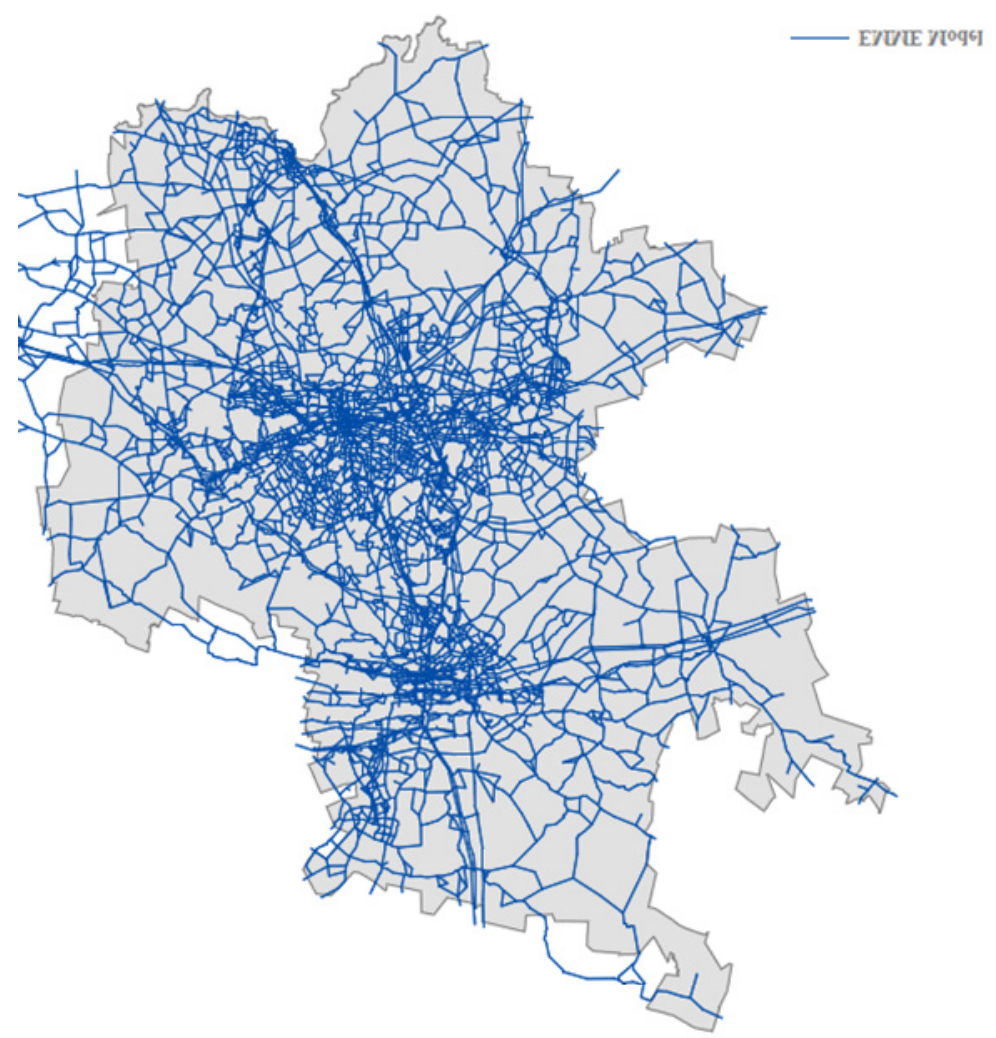

Figure 14: Regional transport model. 


\section{CONCLUDING COMMENTS}

Transportation modelling and simulation as interface in the planning process plays an important role in enabling spatial development. The models developed by transport planning professionals are simplified representations of reality.

Transportation modelling is a young and developing science that has generally developed as part of the evolution of advancing computer technology and was originally developed to solve highway capacity problems. Many mistakes have been made in the past where inappropriate solutions were implemented - with hind sight. A classic mistake includes where new infrastructure provision is considered purely on the basis of the "predict and provide" approach resulting in infrastructure being implemented completely surplus to requirements and unnecessary. The consequence is an unpractical and unaffordable cost to government.

However, although mistakes have been made in the past, models have also proven their worth in solving highly complex problems and the current diverse range of modelling software available means that models are becoming more bespoke in how they support traffic engineers and transportation modellers addressing specific problems.

Transportation models should be seen for what they are: a support tool that helps in decision making. They will always require humans to contextualise and interpret outputs, considering the results in context of specific environments with unique characteristics, features, balancing needs, different stakeholder opinions and humans occupying and living in space.

Over time the relationship between transportation and land use has been better understood. Transport design more often than not determines the success of urban environments and can either be an enabling factor or a hindrance. Transportation modelling should therefore always be considered as a tool that supports decisions and not the panacea to every traffic - transport - land use problem we face or wish to solve. It is expected that transportation modelling will diversify and develop significantly more sophisticated techniques to simulate human behaviour and hence transport flow in the decades to come to better support decision making in planning.

\section{REFERENCES}

[1] Goulias, K.G., Travel behavior and demand analysis and prediction, University of California, 65 pp., 2007.

[2] Tri-County Regional Planning Commission, Travel forecast model development, 14 pp., 2009.

[3] American Association of State Highway and Transportation Officials, Handbook on integrating land use considerations into transportation projects to address induced growth, 52 pp., 2005.

[4] Toth, G., Integrating land use and transportation planning through placemaking. Projects for public space, Online. http://www.pps.org/blog/integrating-land-use-andtransportation-planning-through-placemaking/

[5] Wegener, M., Overview of land use transport models. Handbook of Transport Geography and Spatial Systems, Vol. 5, Handbooks in Transport, eds D.A. Hensher \& K.J. Button, Elsevier: Amsterdam, pp. 127-146, 2004.

[6] United States Department of Transportation Federal Highway Authority of Transportation, Planning for transportation in rural areas, Online. http://www.fhwa.dot.gov/planning/publications/rural_areas_planning/page06.cfm, 2012. 
[7] United States Department of Transportation Federal Highway Administration, The transportation planning process: key issues, 77 pp., 2007, Online. https://www.planning.dot.gov/documents/briefingbook/bbook.htm

[8] Victoria Transport Policy Institute, TDM Encyclopaedia. Transport model improvements improving methods for evaluating the effects and value of transportation system changes, 2014, Online. http://www.vtpi.org/tdm/tdm125.htm

[9] Litman, T., Understanding transport demands and elasticities: How prices and other factors affect travel behaviour, Victoria Transport Policy Institute, 76 pp., 2013.

[10] Chandrasekaran, B., Survey of network traffic models, Online. http://www.cse.wustl.edu/ jain/cse567-06/traffic_models3.htm

[11] Florian, M., Models and software for urban and regional transportation planning, Centre for Research on Transportation, 2008.

[12] Brockwell, A.E., Chan, N.H. \& Lee, P.K., A class of models for aggregated traffic volume time series, 17 pp., 2008.

[13] Jha, K., Sinha, N., Arkatkar, S.S. \& Sarkar, A.K., Modelling growth trend and forecasting techniques for vehicular population in India. International Journal for Traffic and Transport Engineering, 3(2), pp. 139-158, 2013.

[14] Meyer, D.M. \& Miller, E.J., Urban Transportation Planning: A Decision-Oriented Approach, McGraw-Hill: USA, 2001.

[15] He, N. \& Zhao, S., Discussion of basic elasticity models applied to induced traffic in China. Proceedings of the Eastern Asia Society for Transportation Studies, 9, 2013.

[16] Boxill, S.A. \& Yu, L., An evaluation of traffic simulation models for supporting ITS development, Center for Transportation Training and Research, Texas Southern University, Houston, 116 pp., 2000.

[17] Department of Transport, Revised South African Trip Generation Rates Manual, 2007.

[18] Committee of Transport Officials, Technical Methods for Highways (TMH) 17, Vol. 1, Trip Data Manual, 84 pp., 2012.

[19] Institute of Transport Engineers (ITE), Trip Generation Manual, 9th ed., 1216 pp., 2012.

[20] Chiu, Y., Bottom, J., Mahut, M., Paz, A., Balakrishna, R., Waller, T. \& Hicks, J., Dynamic Traffic Assignment, Transportation Network Modelling Committee, Transportation Research Board, 62 pp., 2011.

[21] United States Department of Transportation Federal Highway Administration, Calibration and Validation, 36 pp., 2009.

[22] Wegmann, F. \& Everett, J., Minimum travel demand model calibration and validation guidelines for the State of Tennessee, University of Tennessee, Center for Transportation Research, 25 pp., 2009. 